\title{
Clinically Different Phenotypes of Obstructive Sleep Apnea according to 24 Hours Heart Rate Tachogram Pattern
}

\author{
Soo Jeon Choi, MD, $\mathrm{PhD}^{1}$, Joung Sook Kim, MD, $\mathrm{PhD}^{2}$ \\ 'Division of Respirology, Department of Medicine, Inje University College of Medicine, Sanggye Paik Hospital, Seoul, \\ ${ }^{2}$ Department of Health Promotion Medicine, Ewha Womans University College of Medicine, Seoul, Korea
}

\begin{abstract}
Background and Objective Heart rate vatiability (HRV) parameters are altered during sleep in patients with obstructive sleep apnea (OSA). Nondipping of nocturnal heart rate during sleep is characteristic, but not all patients with OSA show this. The aim of this study was to identify differences in OSA patients with or without nocturnal heart rate dipping.

Methods We retrospectively reviewed patients $(n=40)$ who were confirmed to have OSA [4\% Oxygen desaturation index (ODI4) > $5 / \mathrm{hr}$ ) by a simplified sleep study and underwent a 24 hours Holter electrocardiogram. The OSA patients were compared with control subjects $(\mathrm{n}=72)$, who had been found to have no significant medical diseases.

Results Most HRV parameters were lowered in OSA patients [standard deviation of all normal R-R intervals (SDNN): 99.23 vs. 149.06 ms: $\mathrm{p}<0.0001$. The OSA patients were divided to two subgroups according to their 24 hours HR tachogram patterns. A HR nondipper subgroup $(\mathrm{n}=18)$ showed a marked decrease in SDNN when compared with a HR dipper subgroup $(\mathrm{n}=22)(\mathrm{SDNN} 71.72 \mathrm{vs.} 121.72 \mathrm{~ms}$ : $\mathrm{p}<0.0001)$. SDNN was not associated with a severity index of OSA (ODI4) $(\mathrm{r}=-0.15, \mathrm{p}=0.3619)$.

Conclusions In the OSA patients, most HRV parameters (SDNN, standard deviation of the average normal R-R intervals for each 5-min period (SDANN), percentage of adjacent cycles that are $>50 \mathrm{~ms}$ apart (pNN50\%), root mean square successive differences in milliseconds were lower than in the control subjects. The OSA patients can be divided to two subgroups according to a 24 hours HR tachogram patterns (HR dipper and nondipper). In HR nondipper OSA subgroup, HRV parameters (SDNN, SDANN), Mean $\mathrm{SaO}_{2}$ and basal heart rate during sleep were markedly lowered, but were not associated with an OSA severity (ODI4).

Sleep Med Res 2011;2:21-26
\end{abstract}

Key Words Obstructive sleep apnea, Heart rate variability, Heart rate dipping, Endophenotype.

Received: October 24, 2010

Revised: October 25, 2010

Accepted: October 26, 2010

Correspondence

Joung Sook Kim, MD, PhD

Department of Health Promotion

Medicine, Ewha Womans University

College of Medicine, Mok 5-dong,

Yangcheon-gu, Seoul 158-710, Korea

Tel +82-2-2650-5922

Fax +82-2-2650-5037

E-mail kjshpc@ewha.ac.kr

\section{INTRODUCTION}

The disappearance of nocturnal physiological heart rate dipping has been observed in patients with obstructive sleep apnea (OSA), and this phenomenon might suggest the existence of underlying OSA. ${ }^{1}$ Nondipping of nocturnal (that means during sleep) heart rate may be due to disturbed sleep or fragmentation of sleep, which is a characteristic of OSA, but not all OSA patients show this tendency. We therefore attempted to identify clinical differences in OSA patients with or without nocturnal heart rate dipping.

\section{METHODS}

We retrospectively reviewed patients $(\mathrm{n}=40$, male 35 , female 5 ) who were confirmed to have OSA [ $4 \%$ Oxygen desaturation index (ODI4) > 5/hr] by Win-Visi monitoring system (Stowood Scientific Instrument, Oxford, UK) and had undergone 24 hours monitoring with a Holter electrocardiogram (Marquette Series 8000 Holter Scanner, Milwaukee, USA). The patients with OSA and a comparison group of control subjects $(n=72$, male 40 , female 32$)$ were recruited from medical records of a tertiary general hospital. The control subjects had been found to have no significant medical diseases. The 24 hours heart rate tachogram pattern was 
taken from a Holter electrocardiogram (Marquette Heart Rate Variability Software Version MARS 4.1B). A heart rate dipper pattern was defined as the dipping of nocturnal heart rate more than $10 \%$ from baseline level before sleep. Heart rate vatiability (HRV) parameters were derived from the Holter monitoring data. Comparison of data between patients with OSA, control subjects and OSA subgroups were done with T test using Med-
Calc 11.4 statistical program (Mariakerke, Belgium).

\section{RESULTS}

As expected, when we compared the parameters of OSA patients with those of the control subjects, OSA patients showed

Table 1. HRV parameters in OSA and control subjects

\begin{tabular}{|c|c|c|c|c|c|}
\hline & \multicolumn{2}{|c|}{ OSA patients $(n=40)$} & \multicolumn{2}{|c|}{ Control subjects $(\mathrm{n}=72)$} & \multirow{2}{*}{$\mathrm{p}$} \\
\hline & Mean & $\mathrm{SD}$ & Mean & SD & \\
\hline Age & 52.60 & 13.32 & 50.32 & 13.46 & 0.3904 \\
\hline MeanNN & 750.28 & 122.58 & 833.47 & 93.60 & 0.0001 \\
\hline SDNN & 99.23 & 35.46 & 149.06 & 36.19 & $<0.0001$ \\
\hline SDANN & 87.40 & 35.85 & 136.47 & 35.46 & $<0.0001$ \\
\hline pNN50\% & 5.21 & 5.53 & 9.50 & 8.45 & 0.0048 \\
\hline rMSSD & 23.30 & 8.89 & 30.79 & 11.80 & 0.0007 \\
\hline LHR & 1.59 & 0.54 & 1.61 & 0.38 & 0.7687 \\
\hline SHR & 72.13 & 15.88 & 58.54 & 8.33 & $<0.0001$ \\
\hline
\end{tabular}

HRV: heart rate vatiability, OSA: obstructive sleep apnea, MeanNN: mean value of all normal-to-normal interbeat intervals, SDNN: standard deviation of all normal R-R intervals, SDANN: standard deviation of the average normal R-R intervals for each 5-min period, pNN50\%: percentage of adjacent cycles that are > $50 \mathrm{~ms}$ apart, rMSSD: root mean square successive differences in milliseconds, LHR: ratio of low to high frequency, SHR: basal heart rate during sleep.

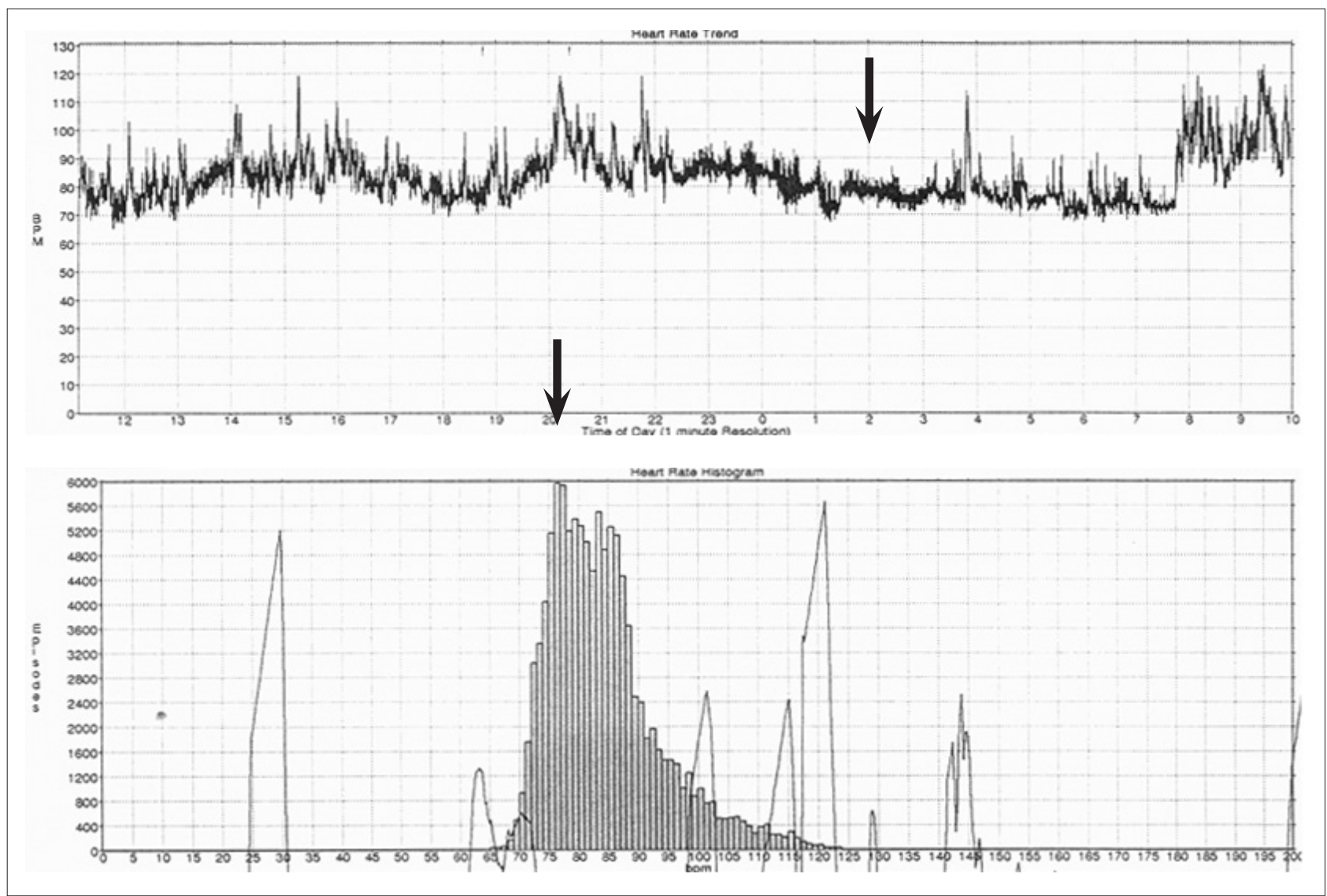

Fig. 1. Heart rate nondipper: male 73-year-old, obstructive sleep apnea syndrome, $4 \%$ Oxygen desatuation index 29 , SDNN 78 ms, sleep heart rate 76 (arrow: Sleep heart rate). 
lower HRV values such as mean value of all normal-to-normal interbeat intervals (Mean NN), standard deviation of all normal R-R intervals (SDNN), standard deviation of the average normal R-R intervals for each 5 -min period (SDANN), percentage of adjacent cycles that are $>50 \mathrm{~ms}$ apart (pNN50\%), root mean square successive differences in milliseconds (rMSSD) (Table 1). The basal heart rate during sleep (SHR) taken from the 24 hours heart rate tachogram also showed a marked differ- ence with the OSA group displaying significantly higher SHR values than the controls (Table 1). When the OSA group was divided into two separate subgroups based on their 24 hours heart rate tachogram pattern (HR nondipper vs dipper) (Figs. 1 and 2$),{ }^{2}$ the HR nondipper subgroup ( $\mathrm{n}=18$ ) was found to be significantly different from the HR dipper subgroup $(n=22)$ in SDNN, SDANN, ratio of low to high frequency (LHR), Mean $\mathrm{SaO}_{2}$, and SHR (Table 2 and 3)(Figs. 3, 4 and 5). The two sub-

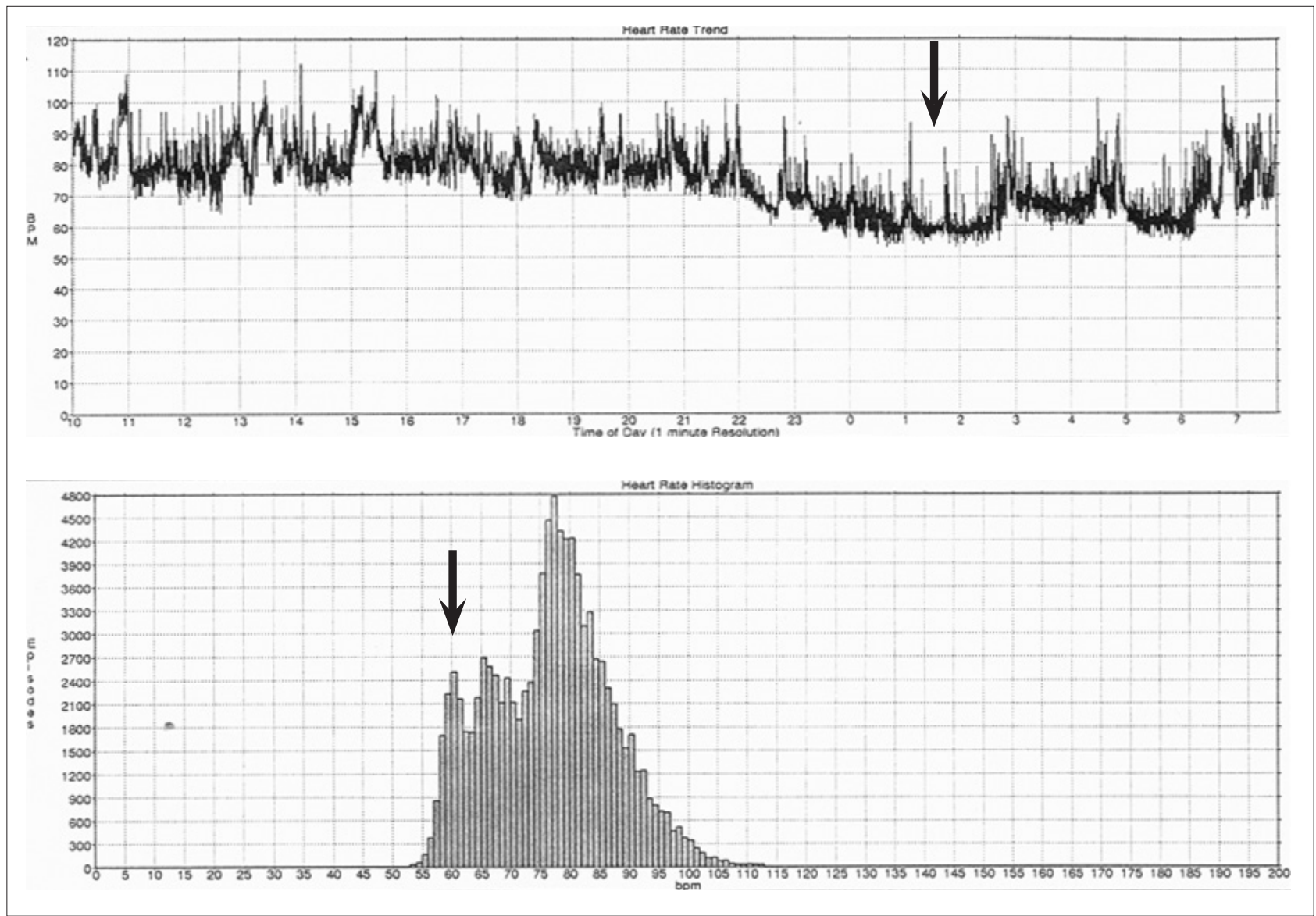

Fig. 2. Heart rate dipper: male 47-year-old, Obstructive sleep apnea syndrome, 4\% Oxygen desatuation index 38.8 , SDNN $111 \mathrm{~ms}$, sleep heart rate 60 (arrow: Sleep heart rate).

Table 2. Differences between HR nondippers $(n=18)$ and dippers $(n=22)$

\begin{tabular}{|c|c|c|c|c|c|}
\hline & \multicolumn{2}{|c|}{ HR nondippers } & \multicolumn{2}{|c|}{ HR dippers } & \multirow{2}{*}{$\mathrm{p}$} \\
\hline & Mean & $\mathrm{SD}$ & Mean & SD & \\
\hline Age & 56.22 & 13.00 & 49.64 & 13.13 & 0.1212 \\
\hline ESS & 11.44 & 4.66 & 10.18 & 3.92 & 0.3577 \\
\hline BMI & 29.67 & 4.51 & 27.32 & 3.98 & 0.0887 \\
\hline NC (inches) & 16.51 & 1.33 & 16.02 & 1.28 & 0.2474 \\
\hline ODI4 & 28.82 & 24.01 & 25.84 & 20.18 & 0.6721 \\
\hline PRR & 31.67 & 15.90 & 38.55 & 16.65 & 0.1926 \\
\hline Mean $\mathrm{SaO}_{2}$ & 90.98 & 4.58 & 93.58 & 1.37 & 0.0158 \\
\hline SHR & 81.56 & 18.39 & 64.41 & 7.48 & 0.0003 \\
\hline
\end{tabular}

HR: heart rate, ESS: Epworth sleepiness scale, BMI: body mass index, NC: neck circumference, ODI4: 4\% oxygen desaturation index, PRR: pulse rate rise, SHR: basal heart rate during sleep. 
groups did not differ in terms of ODI4, pulse rate rise (PRR) $>6$ / min, Epworth sleepiness scale, Body mass index, or neck circumference (Table 2 and 3). The results of a correlation analysis are shown in Table 4. In the undifferentiated OSA group, SD$\mathrm{NN}$ was found to be significantly related to both SHR and Mean $\mathrm{SaO}_{2}$, but it was not associated with Mean $\mathrm{SaO}_{2}$ in the HR dipper subgroup. SHR was related to most HRV parameters (Mean $\mathrm{NN}, \mathrm{SDNN}, \mathrm{SDANN}$, pNN50\%, and rMSSD) in patients with

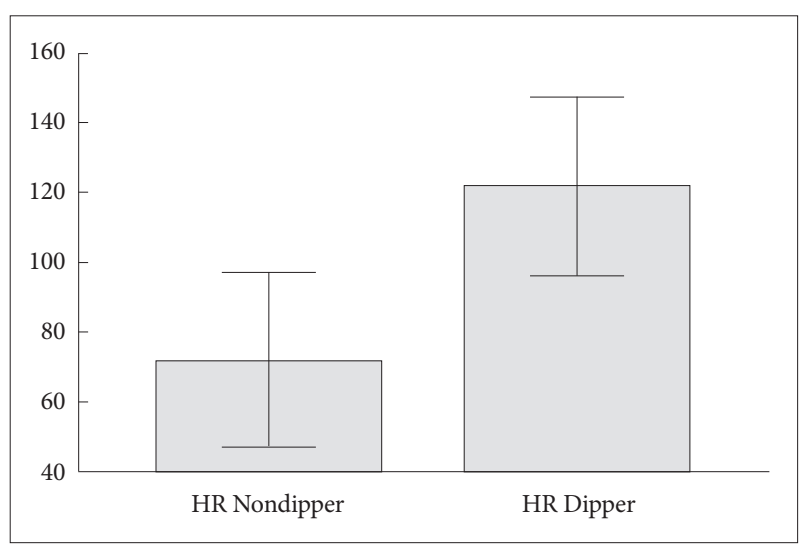

Fig. 3. Standard deviation of all normal R-R intervals $(\mathrm{ms})$ in heart rate nondipper and dipper (mean + SD) $(71.72+25.01$ vs. 121.73 $+25.48, p<0.0001)$.

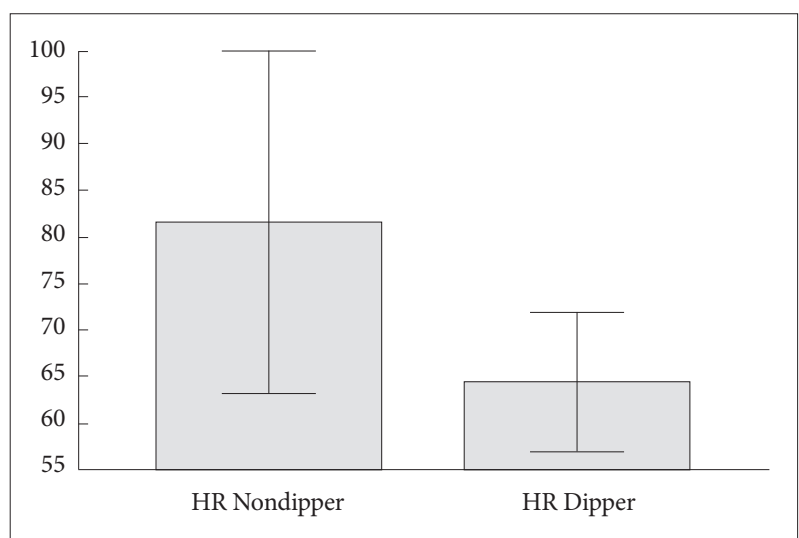

Fig. 4. Sleep heart rate (/min) in HR Nondipper and Dipper (mean + $\mathrm{SD})(81.56+18.39$ vs. $64.41+7.48, p=0.0003)$.
OSA, but in the HR dipper subgroup, it was unrelated to rMSSD, pNN50, ODI4, PRR and Mean $\mathrm{SaO}_{2}$. In contrast, SHR was significantly associated with ODI4, PRR, Mean $\mathrm{SaO}_{2}, \mathrm{MeanNN}$, SDNN, SDANN, pNN50\%, and rMSSD in the HR nondipper subgroup.

\section{DISCUSSION}

Almost all energy on earth is derived from the sun. On our planet, biological systems have evolved to develop sophisticated and efficient processes of energy utilization. ${ }^{3-6}$ Efficiency of energy utilization is reflected on the interaction of heart and lung, or 'cardiopulmonary coupling. We can detect and measure the efficiency of cardiopulmonary coupling in many ways. HRV analysis is one well known method of evaluating cardiopulmonary coupling. HRV is known to be decreased in OSA, ${ }^{7}$ but increased HRV in OSA has been reported. ${ }^{8,9}$ The HRV pattern during sleep has revealed pathological, as well as physiological changes. The pathological changes induced by sleep disordered breathing may therefore increase HRV parameters, especially in severe OSA. ${ }^{8,9}$ A daytime decrease in HRV may reflect sympathetic overactivity as a carry-over effect, so the 24 hours HRV pattern is more important than night time HRV. Contrary to a

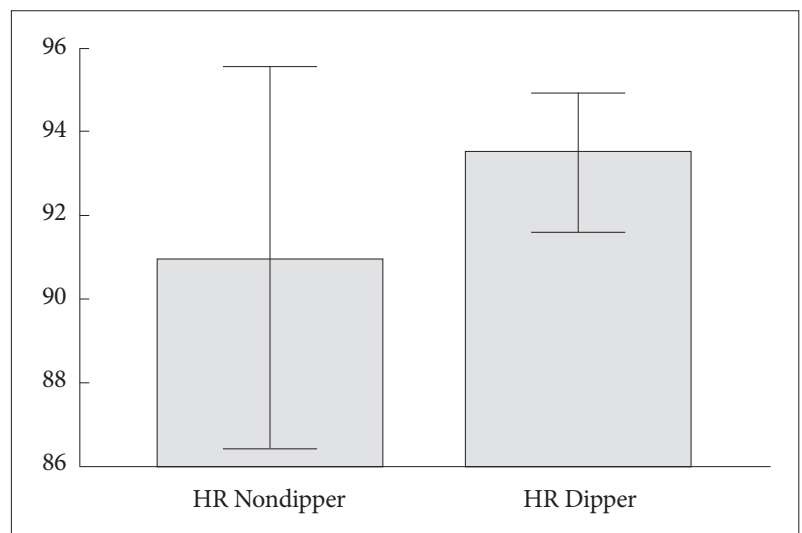

Fig. 5. Mean $\mathrm{SaO}_{2}$ in heart rate nondipper and dipper (mean + SD) $(90.98+4.58$ vs. $93.58+1.37, p=0.0158)$.

Table 3. HRV parameters in HR nondippers $(n=18)$ and dippers $(n=22)$

\begin{tabular}{|c|c|c|c|c|c|}
\hline & \multicolumn{2}{|c|}{ HR nondippers } & \multicolumn{2}{|c|}{ HR dippers } & \multirow{2}{*}{$\mathrm{p}$} \\
\hline & Mean & SD & Mean & SD & \\
\hline MeanNN & 744.83 & 148.93 & 754.73 & 99.52 & 0.8033 \\
\hline SDNN & 71.72 & 25.02 & 121.73 & 25.48 & $<0.0001$ \\
\hline SDANN & 59.72 & 20.89 & 110.05 & 28.97 & $<0.0001$ \\
\hline pNN50\% & 5.66 & 6.06 & 4.846 & 4.55 & 0.6507 \\
\hline rMSSD & 23.22 & 9.90 & 23.36 & 8.22 & 0.9609 \\
\hline LHR & 1.38 & 0.45 & 1.76 & 0.55 & 0.0260 \\
\hline
\end{tabular}

HRV: heart rate vatiability, MeanNN: mean value of all normal-to-normal interbeat intervals, SDNN: standard deviation of all normal R-R intervals, SDANN: standard deviation of the average normal R-R intervals for each 5-min period, pNN50\%: percentage of adjacent cycles that are $>50 \mathrm{~ms}$ apart, rMSSD: root mean square successive differences in milliseconds, LHR: ratio of low to high frequency. 
previous report, ${ }^{7}$ the severity index of OSA (ODI4) was not associated with SDNN, which is the best index of $\operatorname{HRV}(\mathrm{r}=$ $-0.15, \mathrm{p}=0.3619)$. Certain other parameters of HRV were not associated with ODI4: these were SDANN, rMSSD, and pNN$50 \%$. ODI4 was related only to MeanNN and LHR. The two sub- groups were different in the association with ODI4 and Mean$\mathrm{NN}$ :, the relationship held in HR nondipper subgroup $(\mathrm{r}=$ -0.57, $\mathrm{p}=0.0142$ ), but not in HR dipper subgroup $(\mathrm{r}=-0.11, \mathrm{p}$ $=0.6150)$. SDNN had significant relationship with Mean $\mathrm{SaO}_{2}$ $(\mathrm{r}=0.56, \mathrm{p}=0.0002)$ in the undifferentiated OSA group, wher-

Table 4. Correlations between SDNN, SHR and ODI4 in HR nondipper and dipper subgroups

\begin{tabular}{|c|c|c|c|c|c|c|c|}
\hline & & \multicolumn{2}{|c|}{ OSA patients $(\mathrm{n}=40)$} & \multicolumn{2}{|c|}{ HR nondipper OSA $(\mathrm{n}=18)$} & \multicolumn{2}{|c|}{ HR dipper OSA $(\mathrm{n}=22)$} \\
\hline & & $\mathrm{r}$ & $\mathrm{p}$ & $\mathrm{r}$ & $\mathrm{p}$ & $\mathrm{r}$ & $\mathrm{p}$ \\
\hline \multirow[t]{8}{*}{ SDNN } & Age & -0.23 & 0.1483 & -0.05 & 0.8325 & -0.10 & 0.6444 \\
\hline & ESS & -0.18 & 0.2548 & -0.43 & 0.0771 & 0.18 & 0.4139 \\
\hline & BMI & -0.27 & 0.0974 & -0.33 & 0.1832 & 0.09 & 0.6797 \\
\hline & $\mathrm{NC}$ & -0.14 & 0.3897 & -0.15 & 0.5642 & 0.10 & 0.6499 \\
\hline & ODI4 & -0.15 & 0.3619 & -0.27 & 0.2859 & -0.02 & 0.9154 \\
\hline & PRR & 0.09 & 0.5864 & -0.23 & 0.3677 & 0.02 & 0.9442 \\
\hline & Mean $\mathrm{SaO}_{2}$ & 0.56 & 0.0002 & 0.69 & 0.0017 & 0.08 & 0.7096 \\
\hline & SHR & -0.81 & $<0.0001$ & -0.81 & $<0.0001$ & -0.74 & 0.0001 \\
\hline \multirow[t]{13}{*}{ SHR } & Age & -0.08 & 0.6333 & -0.36 & 0.1426 & -0.14 & 0.523 \\
\hline & ESS & 0.27 & 0.0921 & 0.41 & 0.0937 & -0.15 & 0.4951 \\
\hline & BMI & 0.26 & 0.1115 & 0.30 & 0.2233 & -0.22 & 0.3238 \\
\hline & $\mathrm{NC}$ & 0.14 & 0.4023 & 0.20 & 0.4186 & -0.28 & 0.203 \\
\hline & ODI4 & 0.35 & 0.0276 & 0.56 & 0.0159 & 0.00 & 0.9862 \\
\hline & PRR & 0.20 & 0.2097 & 0.66 & 0.0029 & -0.02 & 0.9382 \\
\hline & Mean $\mathrm{SaO}_{2}$ & -0.70 & $<0.0001$ & -0.73 & 0.0005 & 0.03 & 0.9084 \\
\hline & MeanNN & -0.75 & $<0.0001$ & -0.94 & $<0.0001$ & -0.73 & 0.0001 \\
\hline & SDNN & -0.81 & $<0.0001$ & -0.81 & $<0.0001$ & -0.74 & 0.0001 \\
\hline & SDANN & 0.76 & $<0.0001$ & -0.75 & 0.0003 & -0.74 & 0.0001 \\
\hline & pNN50\% & -0.34 & 0.034 & -0.51 & 0.0304 & -0.32 & 0.1482 \\
\hline & rMSSD & -0.44 & 0.0047 & -0.63 & 0.005 & -0.34 & 0.1232 \\
\hline & LHR & -0.11 & 0.5088 & 0.00 & 0.9881 & 0.32 & 0.1522 \\
\hline \multirow[t]{13}{*}{ ODI4 } & Age & -0.54 & 0.0003 & -0.66 & 0.0029 & -0.50 & 0.0176 \\
\hline & ESS & 0.06 & 0.7154 & 0.31 & 0.2183 & -0.24 & 0.2781 \\
\hline & BMI & 0.25 & 0.1128 & 0.43 & 0.072 & 0.04 & 0.8582 \\
\hline & $\mathrm{NC}$ & 0.32 & 0.042 & 0.40 & 0.1005 & 0.23 & 0.2928 \\
\hline & PRR & 0.76 & $<0.0001$ & 0.82 & $<0.0001$ & 0.79 & $<0.0001$ \\
\hline & Mean $\mathrm{SaO}_{2}$ & -0.40 & 0.0112 & -0.37 & 0.1343 & -0.68 & 0.0005 \\
\hline & MeanNN & -0.38 & 0.0155 & -0.57 & 0.0142 & -0.11 & 0.615 \\
\hline & SDNN & -0.15 & 0.3619 & -0.27 & 0.2859 & -0.02 & 0.9154 \\
\hline & SDANN & -0.13 & 0.4222 & -0.22 & 0.3895 & -0.05 & 0.8174 \\
\hline & pNN50\% & -0.19 & 0.2362 & -0.19 & 0.4468 & -0.21 & 0.3503 \\
\hline & rMSSD & -0.26 & 0.1100 & -0.26 & 0.2987 & -0.25 & 0.2549 \\
\hline & LHR & 0.42 & 0.0067 & 0.40 & 0.1024 & 0.56 & 0.0072 \\
\hline & SHR & 0.35 & 0.0276 & 0.56 & 0.0159 & 0.00 & 0.9862 \\
\hline
\end{tabular}

SDNN: standard deviation of all normal R-R intervals, SHR: basal heart rate during sleep, ODI4: 4\% oxygen desaturation index, HR: heart rate, ESS: Epworth sleepiness scale, BMI: body mass index, NC: neck circumference, PRR: pulse rate rise, MeanNN: mean value of all normal-tonormal interbeat intervals, SDANN: standard deviation of the average normal R-R intervals for each 5-min period, pNN50\%: percentage of adjacent cycles that are >50 ms apart, rMSSD: root mean square successive differences in milliseconds, LHR: ratio of low to high frequency, SHR: basal heart rate during sleep. 
eas this relationship was found in the HR nondipper subgroup $(\mathrm{r}=0.69, \mathrm{p}=0.0017)$. In the HR dipper subgroup, there was no association with Mean $\mathrm{SaO}_{2}(\mathrm{r}=0.08, \mathrm{p}=0.7096)$. This finding may suggest the possibility of different prognoses, but a long term follow study will be necessary to prove this is the case. It may be thought that in the HR dipper subgroup, better SDNN (121.73 vs. $71.72 \mathrm{~ms}$ ), Mean $\mathrm{SaO}_{2}$ (93.58 vs. 90.98\%) and lower SHR (64.41 vs. $81.56 / \mathrm{min}$ ) already suggested a more preserved homeostatic condition. SDNN showed the highest association with SHR ( $\mathrm{r}=-0.81, \mathrm{p}<0.0001)$ in both the subgroups of HR nondipper and dipper. SDNN is a very sensitive predictor of morbidity and mortality, ${ }^{10}$ and resting heart rate is strongly related to the risk of sudden cardiac death. ${ }^{11}$ It is therefore not surprising that SDNN was closely related to SHR in our study ( $\mathrm{r}$ $=-0.81, \mathrm{p}<0.0001)$. This may be deduced from the finding that HR and SDNN have shown reciprocal changes simulating mirror images. ${ }^{12}$ The division of OSA patients into HR dipper and nondipper subgroups, which is very simple and easy by 24 hours heart rate tachogram, showed marked differences in SDNN, SDANN, SHR and $\mathrm{MeanSaO}_{2}$, but no differences in ODI4 and PRR. The nocturnal HR dipping pattern had no association with ODI4 or, PRR. This finding may suggest the possibility that HR dipping during sleep is a kind of endophenotype. ${ }^{13}$

In the long term, many of the HRV parameters (SDNN, SDANN, SHR and Mean $\mathrm{SaO}_{2}$ ) are expected to be related to cardiovascular mortality and morbidity, but further studies will be necessary to confirm this. Our study suggest that the 24 hours heart rate tachogram pattern may give clinically significant informations about subgroup differentiation (HR dipper vs. nondipper) and resting basal heart rate (i.e., sleep heart rate: SHR), which showed markedly different HRV pattern.

Limitations. This was not prospective study, and sampling biases are therefore a particular risk. Moreover the diagnosis of OSA was confirmed by a simplified sleep study (Win-Visi monitoring system), and the results of this may be different from those using traditional polysomnography.

\section{Conclusion}

In OSA patients, most HRV parameters (SDNN, SDANN, pNN$50 \%$, and $\mathrm{rMSSD}$ ) showed lower levels than in the control sub- jects. When OSA patients were divided to two subgroups according to 24 hours HR tachogram patterns (HR dipper vs. nondipper), HRV parameters (SDNN, SDANN, Mean $\mathrm{SaO}_{2}$, and SHR) in the HR nondipper subgroups were markedly lowered, but were not associated with the OSA severity index of ODI4.

\section{Conflicts of Interest}

The authors have no financial conflicts of interest.

\section{REFERENCES}

1. Garrigue S, Bordier P, Barold SS, Clementy J. Sleep apnea: a new indication for cardiac pacing? Pacing Clin Electrophysiol 2004;27:204-11.

2. Choi SJ, Choi SB. Clinical feature of heart rate variability in obstructive sleep apnea patients. Korean J Sleep Med 2006;8:14-9.

3. Porges SW. Orienting in a defensive world: mammalian modifications of our evolutionary heritage. A Polyvagal Theory. Psychophysiology 1995; 32:301-18.

4. Porges SW. The polyvagal theory: phylogenetic substrates of a social nervous system. Int J Psychophysiol 2001;42:123-46.

5. Yun AJ, Lee PY, Doux JD, Conley BR. A general theory of evolution based on energy efficiency: its implications for diseases. Med Hypotheses 2006;66:664-70.

6. Yun AJ, Lee PY, Doux JD. A paradigm for viewing biologic systems as scale-free networks based on energy efficiency: implications for present therapies and the future of evolution. Med Hypotheses 2006;67:651-7.

7. Aydin M, Altin R, Ozeren A, Kart L, Bilge M, Unalacak M. Cardiac autonomic activity in obstructive sleep apnea: time-dependent and spectral analysis of heart rate variability using 24-hour Holter electrocardiograms. Tex Heart Inst J 2004;31:132-6.

8. Yang A, Schäfer H, Manka R, Andrié R, Schwab JO, Lewalter T, et al. Influence of obstructive sleep apnea on heart rate turbulence. Basic Res Cardiol 2005;100:439-45.

9. Szollosi I, Krum H, Kaye D, Naughton MT. Sleep apnea in heart failure increases heart rate variability and sympathetic dominance. Sleep 2007; 30:1509-14.

10. Karcz M, Chojnowska L, Zareba W, Ruzyłł W. Prognostic significance of heart rate variability in dilated cardiomyopathy. Int J Cardiol 2003; 87:75-81.

11. Jouven X, Empana JP, Schwartz PJ, Desnos M, Courbon D, Ducimetière P. Heart-rate profile during exercise as a predictor of sudden death. N Engl J Med 2005;352:1951-8.

12. Vandewalle G, Middleton B, Rajaratnam SM, Stone BM, Thorleifsdottir B, Arendt J, et al. Robust circadian rhythm in heart rate and its variability: influence of exogenous melatonin and photoperiod. J Sleep Res 2007;16:148-55

13. Thayer JF, Lane RD. Claude Bernard and the heart-brain connection: further elaboration of a model of neurovisceral integration. Neurosci Biobehav Rev 2009;33:81-8. 